\title{
Why One Anastomosis (Mini-) Gastric Bypass May Cause Less Immediate Dumping than Roux- En-Y Gastric Bypass: An Opinion
}

\author{
Jacques M Himpens* \\ Bariatric-metabolic surgery unit, Belgium
}

\section{ISSN: 2637-7632}

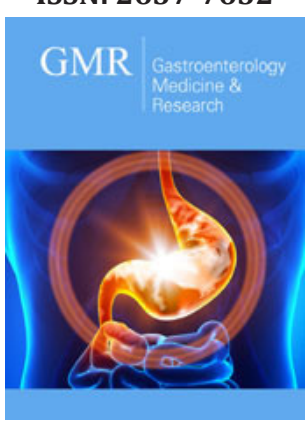

*Corresponding author: Jacques M Himpens, Bariatric-metabolic surgery unit, The CHIREC hospitals, Brussels and Braine-l'Alleud, Attending surgeon, St Pierre University Hospital, Brussels, Belgium

Submission: 留June 28, 2019

Published: 制July 02, 2019

Volume 3 - Issue 3

How to cite this article: Jacques $M$ Himpens, Why One Anastomosis (Mini-) Gastric Bypass May Cause Less Immediate Dumping than Roux-En-Y Gastric Bypass: An Opinion. Gastro Med Res. 3(3). GMR.000563. 2019.

DOI: 10.31031/GMR.2019.03.000563

Copyright@ Jacques M Himpens, This article is distributed under the terms of the Creative Commons Attribution 4.0 International License, which permits unrestricted use and redistribution provided that the original author and source are credited.

\section{Introduction}

The laparoscopic one anastomosis (mini-) gastric bypass (OAGB) is quickly gaining popularity as a bariatric-metabolic operation. One of the main reasons of the increase in popularity is - without any doubt- the technical ease of the procedure. However, there are other possible benefits that may help explain the increasing numbers. One of the benefits may be the alleged reduction of incidence and importance of the immediate dumping syndrome, which is still considered one of the major drawbacks of the gastric bypass [1]. Nowadays, the difference between the Roux-en-Y (RYGB) and OAGB tends to be limited to the interposition of a $60 \mathrm{~cm}$ long alimentary limb in the former, whereas this limb, by definition, does not exist in the latter. Indeed, "modern" versions of the RYGB imply a long $(150 \mathrm{~cm})$ biliopancreatic limb -which happens to be the recommended length of the biliopancreatic limb in the OAGB-, and a rather short $(60 \mathrm{~cm})$ alimentary limb [2]. Even the gastric pouch of the RYGB more and more resembles the pouch of the OAGB, as surgeons make the pouch longer and skinnier, and certainly perform a rather wide gastro-enterostomy [3].

In order to investigate the possible differences in glucose metabolism between OAGB and RYGB we analyzed in a lab environment if indeed the oscillations in plasma glucose did vary between the 2 bypass types [4]. To this purpose we observed 3 different groups of patients: 2 groups of patients formerly suffering from morbid obesity, who had undergone either type of bypass, and who had come to an equilibrium in terms of weight loss (i.e. some 3 years postoperatively), and 1 group of healthy volunteers without obesity. We submitted all participants to an oral glucose tolerance test (OGTT) and measured the plasma glucose progression as well as the plasma insulin over a 3 hours period. In addition, we submitted the same participants to an intravenous glucose tolerance test to evaluate the pancreatic reaction independently from the intestinal glucose absorption.

To our surprise, we found that the simple incorporation of a rather short alimentary limb did not at all interfere with the plasma glucose progression in this experimental setting. In fact, the curve of plasma glucose after OAGB appeared to be exactly superimposable to the curve after RYGB. However, it seemed that the progression of plasma insulin differed. There was a tendency (not reaching statistical significance) for higher insulin levels early on in the OAGB group. This resulted in a greater insulinogenic index in the latter group. In other words, there is some indication that the glucose levels across the 2 bypass groups can only be kept identical at the cost of a higher early insulin production in the OAGB group.

What is the mechanism behind this change in insulin production?

We know from the work of Professor Pattou's team [5] that intestinal glucose absorption requires the presence of Sodium in order to activate the Sodium-Glucose Transferase 2 (SGLT2) enzyme. Usually, and certainly in our experiment, the alimentary limb in the RYGB subjects does not contain any salt (because there is no bile in the alimentary limb), unlike in the OAGB subjects, where the absorbed food stuffs (i.e. pure glucose in our experimental setting) come immediately in contact with the bile Sodium, thus allowing the immediate absorption of glucose. In the RYGB patients glucose absorption is just delayed some-the time required for the glucose to progress to the common limb, that contains plenty of bile, hence 
Sodium. The delay in eliciting the insulin response to the glucose absorption however seems to influence the insulin production and to suffice to substantially reduce the insulinogenic index.

But should not the fact that glucose apparently is less absorbed in RYGB mean that the immediate dumping syndrome should be less pronounced after this procedure than after OAGB?

We believe that another mechanism (other than glucose absorption) may play a role in this particular issue. In fact, whereas the delayed dumping syndrome actually is the clinical manifestation of hypoglycemia [6] -which in RYGB and OAGB equally occurs beyond the first hour after food intake-, the early dumping is in fact an osmotic phenomenon [7]. When we go back to our experiment and focus on what actually happens in the alimentary limb after ingestion of a meal consisting of liquid glucose, we may find a likely explanation. In RYGB, no glucose absorption occurs at the level of the alimentary limb. Therefore, the foodstuffs concentration, especially carbohydrates (glucose in our study) remains unchanged in the alimentary limb. Because of their osmotic power, foodstuffs drag fluid into that limb, hereby fulfilling the clinical conditions of early dumping. On the contrary, after OAGB, because of the very active glucose absorption at the level of the alimentary limb, the osmotic potential of the food stuffs (i.e. glucose in our study) should be far less than after RYGB, hence elicit much less of a fluid drag, i.e. less of an early dumping reaction. As mentioned earlier, the clinical finding that OAGB causes less immediate dumping than RYGB has not (yet) been proven, hence a biochemical explanation of this alleged phenomenon is nothing but conjectural. We obviously need more research, and preferably randomized controlled clinical trials to elucidate this important issue. However, the hypothesis may help us understand the possible subtle physiologic differences that exist between the 2 types of bypass and possibly orient the surgeons' (and the patients') preference for one type over another.

\section{References}

1. Emous M, Wolffenbuttel BHR, Totté E, van Beek AP (2017) The shortto mid-term symptom prevalence of dumping syndrome after primary gastric-bypass surgery and its impact on health-related quality of life. Surg Obes Relat Dis 13(9): 1489-1500.

2. Nergaard BJ, Leifsson BG, Hedenbro J, Gislason H (2014) Gastric bypass with long alimentary limb or long pancreato-biliary limb-long-term results on weight loss, resolution of co-morbidities and metabolic parameters. Obes Surg 24(10): 1595-1602.

3. Riccioppo D, Santo MA, Rocha M, Buchpiguel CA, Diniz MA, et al. (2018) Small-volume, fast-emptying gastric pouch leads to better long-term weight loss and food tolerance after Roux-en-Y gastric bypass. Obes Surg 28(3): 693-701.

4. Himpens JM, Vilallonga R, Cadière GB, Leman G (2016) Metabolic consequences of the incorporation of a Roux limb in an omega loop (mini) gastric bypass: Evaluation by a glucose tolerance test at mid-term follow-up. Surg Endosc 30(7): 2935-2945.

5. Baud G, Daoudi M, Hubert T, Raverdy V, Pigeyre M, et al. (2016) Bile diversion in Roux-en-Y gastric bypass modulates sodium-dependent glucose intestinal uptake. Cell Metab 23(3): 547-553.

6. Rogers AM (2019) Prevalence and pathophysiology of early dumping in patients after primary Roux-en-Y gastric bypass during a mixed-meal tolerance test. Surg Obes Relat Dis 15(1): 73-81.

7. Hasler WL (2002) Dumping syndrome. Curr Treat Options Gastroenterol 5(2): 139-145. 